\title{
Extended Hybrid Model Reference Adaptive Control of Piecewise Affine Systems
}

\author{
Mario di Bernardo *, Umberto Montanaro ${ }^{\dagger, *}$, Romeo Ortega ${ }^{\ddagger}$, \\ and Stefania Santini*
}

\begin{abstract}
This note presents an extension to the adaptive control strategy presented in [1] able to counter eventual instability due to disturbances at the input of an otherwise $\mathcal{L}_{2}$ stable closed-loop system. These disturbances are due to the presence of affine terms in the plant and reference model. The existence of a common Lyapunov function is used to prove global convergence of the error system, even in the presence of sliding solutions, as well as boundedness of all the adaptive gains.
\end{abstract}

\section{Introduction}

As notably highlighted in [2], adaptive control of switched systems is still an open problem. Recently, a novel model reference adaptive strategy has been presented in [1] that allows the control of multi-modal piecewise linear (PWL) plants. Specifically, a hybrid model reference adaptive strategy was proposed able to make a PWL plant track the states of an LTI or PWL reference model even if the plant and reference model do not switch synchronously between different configurations. While stability is guaranteed for PWL systems, for affine systems the presence of a non-square integrable disturbance term in the error equations and the possible occurrence of sliding solutions can render the proof of stability inadequate.

${ }^{*}$ M. di Bernardo, U. Montanaro and S. Santini are with the Department of Electrical Engineering and Information Technology, University of Naples Federico II, Italy. E-mail: \{mario.dibernardo, umberto.montanaro, stefania.santini\}@unina.it.

${ }^{\dagger} \mathrm{U}$. Montanaro is with the Department of Industrial Engineering, University of Naples Federico II, Italy. E-mail: umberto.montanaro@unina.it.

${ }^{\ddagger}$ R. Ortega is with the Laboratoire de Signaux et Systemes (SUPELEC), Paris, France. E-mail: Romeo.Ortega@lss.supelec.fr. 
We wish to emphasize that the problem of large state excursions and instabilities caused by constant input disturbances on the closed loop system is a common problem of adaptive control systems seldom highlighted in the literature (see for example [3], [4], and [5] Sec. 4.4.4 p. 173). Indeed, adaptive systems can be represented as the negative feedback interconnection of a passive system (defined by the estimator) and a strictly positive real (SPR) transfer function. A simple application of the passivity theorem establishes that the overall system is $\mathcal{L}_{2}$-stable. However, this property does not ensure that the system will remain stable in the presence of external disturbances which are not $\mathcal{L}_{2}$. A simple example of this scenario is the following system [6]:

$$
\begin{aligned}
& \dot{x}_{1}=f_{1}\left(x_{1}, x_{2}\right)=-x_{1} x_{2} \cos x_{2} \\
& \dot{x}_{2}=f_{2}\left(x_{1}, x_{2}\right)=x_{1}^{2} \cos x_{2}-x_{2}+\pi,
\end{aligned}
$$

where the presence of the constant input $\pi$ can cause exponentially growing trajectories that can significantly disrupt performance.

The aim of this note is to present a modification of the control strategy presented in [1 able to guarantee asymptotic stability of the closed loop system even in the presence of sliding mode trajectories and bounded $\mathcal{L}_{\infty}$ perturbations due to the affine terms in the description of the plant and/or reference model. The idea is to add an extra switching action to the controller in 1 able to compensate the presence of such a disturbance. The proof of stability is obtained by defining an appropriate common Lyapunov function and analyzing its properties along the closed-loop system trajectories within each of the phase space regions where the plant and reference model are characterized by different modes, and along their boundaries. We show that, even in the presence of possible sliding mode trajectories, the origin of the closed-loop error system is rendered asymptotically stable by the extended strategy presented in this paper. A preliminary version of the algorithm suitable to control bimodal piecewise affine system can be found in [7], [8], while experimental validation results are reported in [9]. A possible extension to discrete-time piecewise-affine (PWA) plants can be found in [10], [11.

\section{Problem statement and definitions}

Assume that the state space $\mathbb{R}^{n}$ is partitioned by some smooth boundaries into $M$ domains, say $\left\{\Omega_{i}\right\}_{i \in \mathcal{M}}$ with $\mathcal{M}=\{0,1, \ldots, M-1\}$ such that $\bigcup_{i=0}^{M-1} \Omega_{i}=\mathbb{R}^{n}$ and, given two generic indexes $i_{1}$ and $i_{2} \in \mathcal{M}$ (with $i_{1} \neq i_{2}$ ), it follows $\Omega_{i_{1}} \cap \Omega_{i_{2}}=\emptyset$. 
Let the plant be described by an $n$-dimensional multi-modal PWA system whose dynamics are given by:

$$
\dot{x}=A_{i} x+B u+B_{i} \quad \text { if } \quad x \in \Omega_{i}, \quad i \in \mathcal{M},
$$

where $x \in \mathbb{R}^{n}$ is the state vector, $u \in \mathbb{R}$ is the scalar input, and the matrices $A_{i}, B, B_{i}(i=0,1, \ldots, M-1)$ are assumed to be in control canonical form, i.e.

$$
A_{i}=\left[\begin{array}{cccc}
0 & 1 & \cdots & 0 \\
0 & 0 & \ddots & \vdots \\
\vdots & \vdots & & 1 \\
a_{i}^{(1)} & a_{i}^{(2)} & \cdots & a_{i}^{(n)}
\end{array}\right], B=\left[\begin{array}{c}
0 \\
0 \\
\vdots \\
b
\end{array}\right], B_{i}=\left[\begin{array}{c}
0 \\
0 \\
\vdots \\
b_{i}
\end{array}\right]
$$

with $b>0$. Note that all entries on the last row of the plant matrices $A_{i}$, $B$ and $B_{i}$ are supposed to be unknown. (Notice that many generic bimodal PWL continuous systems can be transformed into such a form as shown in [12].)

The problem is to find an adaptive piecewise feedback law $u(t)$ to ensure that the state variables of the plant track asymptotically the states, say $\widehat{x}(t)$, of a reference model independently from their initial conditions.

Here, we assume that the reference model can be either an LTI system, or a multi-modal PWA system:

$$
\dot{\hat{x}}=\widehat{A}_{\hat{i}} \widehat{x}+\widehat{B} r+\widehat{B}_{\widehat{i}} \quad \text { if } \quad \widehat{x} \in \widehat{\Omega}_{\widehat{i}}, \quad \widehat{i} \in \widehat{\mathcal{M}}
$$

where the state $\widehat{x} \in \mathbb{R}^{n}, \widehat{\mathcal{M}} \triangleq\{0,1, \ldots, \widehat{M}-1\},\left\{\widehat{\Omega}_{\widehat{i}}\right\}_{\widehat{i} \in \widehat{\mathcal{M}}}$ is a partition of $\mathbb{R}^{n}$ into $\widehat{M}$ domains obtained by some smooth boundaries and $r \in \mathbb{R}$ is the input to the reference model. Note that the reference model may possess a number of modes different from the one of the plant, $\widehat{M} \neq M$. Furthermore, we assume that the reference model defined as in (3) is chosen so as not to exhibit sliding solutions and that it is well-posed given the initial condition $\widehat{x}(0)=\widehat{x}_{0}$. In many practical cases, the aim of the control action can be that of compensating the discontinuous nature of the plant. In these situations, the control design presented above offers a simple and viable solution for this to be achieved by simply choosing a smooth or smoother reference model. This often corresponds to the conventional choice of an asymptotically stable LTI reference model in the case of smooth systems.

As for the plant, the matrices of the reference model are chosen to be in the companion form given by $(\widehat{i}=0,1, \ldots, \widehat{M}-1)$ : 


$$
\widehat{A}_{\hat{i}}=\left[\begin{array}{cccc}
0 & 1 & \cdots & 0 \\
0 & 0 & \ddots & \vdots \\
\vdots & \vdots & & 1 \\
\widehat{a}_{\widehat{i}}^{(1)} & \widehat{a}_{\widehat{i}}^{(2)} & \cdots & \widehat{a}_{\widehat{i}}^{(n)}
\end{array}\right], \widehat{B}=\left[\begin{array}{c}
0 \\
0 \\
\vdots \\
\widehat{b}
\end{array}\right], \widehat{B}_{\hat{i}}=\left[\begin{array}{c}
0 \\
0 \\
\vdots \\
\widehat{\hat{b}_{\hat{i}}}
\end{array}\right]
$$

with $\widehat{b}>0$.

In what follows, we use the standard notation in [13] (also adopted in [14]), for both the switching instants of the plant and reference model. More precisely, the switching sequence of the plant is given by:

$$
\Sigma=\left\{x_{0},\left(i_{0}, t_{0}\right),\left(i_{1}, t_{1}\right),\left(i_{2}, t_{2}\right) \ldots\left(i_{p}, t_{p}\right) \ldots \mid i_{p} \in \mathcal{M}, p \in \mathbb{N}\right\},
$$

where $t_{0}=0$ is the initial time instant and $x_{0}$ is the initial state. Note that, as in [13], when $t \in\left[t_{p} ; t_{p+1}\right)$, then $x(t)$ belongs to $\Omega_{i_{p}}$ by definition and, thus, the $i_{p}$-th subsystem is active. Obviously, the switching sequence $\Sigma$ may be finite or infinite. If there is a finite number of switchings, say $p$, then we set $t_{p+1}=\infty$.

For any $j \in \mathcal{M}$, we denote the sequence of switching times when the $j$-th subsystem is switched on as:

$$
\Sigma / j=\left\{t_{j_{1}}, t_{j_{2}}, \ldots t_{j_{s}}, \ldots \mid i_{j_{s}}=j \text { and } s \in \mathbb{N}\right\},
$$

and, thus, the endpoints of the time intervals when the $j$-th subsystem is active can be given as:

$$
\left\{t_{j_{1}+1}, t_{j_{2}+1}, \ldots, t_{j_{s}+1}, \ldots \mid i_{j_{s}}=j \text { and } s \in \mathbb{N}\right\} .
$$

Analogously, we define the switching sequence of the reference model as:

$$
\widehat{\Sigma}=\left\{\widehat{x}_{0},\left(\widehat{i}_{0}, \widehat{t}_{0}\right),\left(\widehat{i}_{1}, \widehat{t}_{1}\right),\left(\widehat{i}_{2}, \widehat{t}_{2}\right) \ldots\left(\widehat{i}_{p}, \widehat{t}_{p}\right) \ldots \mid \widehat{i}_{p} \in \widehat{\mathcal{M}}, p \in \mathbb{N}\right\},
$$

with $\widehat{t}_{0}=0$. Hence, when $t \in\left(\widehat{t}_{p} ; \widehat{t}_{p+1}\right)$ then $\widehat{x}(t) \in \widehat{\Omega}_{\widehat{i}_{p}}$ by definition and the $\widehat{i}_{p}$-th subsystem is active.

For any $\widehat{j} \in \widehat{\mathcal{M}}$ the sequence of switching times when the $\widehat{j}$-th subsystem of the reference model is switched on can be analogously defined as:

$$
\widehat{\Sigma} / \widehat{j}=\left\{\widehat{t}_{\widehat{j}_{1}}, \widehat{t}_{\hat{j}_{2}}, \ldots \widehat{t}_{\widehat{j}_{s}}, \ldots \mid \widehat{i}_{\widehat{j}_{s}}=\widehat{j}, s \in \mathbb{N}\right\}
$$

with the endpoints of the intervals where the $\widehat{j}$-th mode is active being:

$$
\left\{\widehat{t}_{\widehat{j}_{1}+1}, \widehat{t}_{\widehat{j}_{2}+1}, \ldots, \widehat{t}_{\widehat{j}_{s}+1}, \ldots \mid \widehat{i}_{\widehat{j}_{s}}=\widehat{j}, s \in \mathbb{N}\right\} .
$$


We define the "switching signals" $\sigma: \mathbb{R}^{+} \mapsto \mathcal{M}$ and $\widehat{\sigma}: \mathbb{R}^{+} \mapsto \widehat{\mathcal{M}}$ as:

$$
\sigma(t)=i \quad \text { if } \quad x(t) \in \Omega_{i}, \quad \widehat{\sigma}(t)=\widehat{i} \quad \text { if } \quad \widehat{x}(t) \in \widehat{\Omega}_{\widehat{i}}
$$

and the indicator functions $\sigma_{i}(t)$ and $\widehat{\sigma}_{\hat{i}}(t)$, as:

$$
\begin{aligned}
& \sigma_{i}(t)=\left\{\begin{array}{l}
1 \text { if } x(t) \in \Omega_{i}, \\
0 \text { elsewhere, }
\end{array}\right. \\
& \widehat{\sigma}_{\widehat{i}}(t)=\left\{\begin{array}{l}
1 \text { if } \widehat{x}(t) \in \widehat{\Omega}_{\widehat{i}}, \\
0 \text { elsewhere, }
\end{array}\right.
\end{aligned}
$$

with $i=0,1, \ldots, M-1$ and $\widehat{i}=0,1, \ldots, \widehat{M}-1$.

Also, $e_{n} \in \mathbb{R}^{n}$ is defined as the basis vector

$$
e_{n}=\left[\begin{array}{llll}
0 & \ldots & 0 & 1
\end{array}\right]^{T} .
$$

\section{Control Strategy}

The control problem described in Section 2 can be solved by means of an extended switched adaptive strategy as described in the rest of this section. The proposed approach extends the work presented in [1 by exploiting an additional adaptive switching control gain to cope with the presence of the bounded piecewise constant input acting on the closed-loop system when the plant and/or reference model are PWA.

Assumption 1 Assume there exists a matrix $P=P^{T}>0$ such that

$$
P \widehat{A}_{\hat{i}}+\widehat{A}_{\hat{i}}^{T} P<0 \quad \widehat{i}=0,1,2, \ldots, \widehat{M}-1 .
$$

Given the above assumption our main result can be stated as follows.

Theorem 1 Consider a PWA plant of the form (1) and a PWA reference model of the form (3). If the dynamic matrices $\widehat{A}_{\hat{i}}$ of the reference model verify Assumption 1, then the piecewise smooth adaptive control law:

$$
u(t)=K_{R}(t) r(t)+K_{F B}(t) x(t)+K_{A}(t),
$$

where

$$
\begin{aligned}
K_{R}(t) & =\alpha \int_{0}^{t} y_{e}(\tau) r(\tau) d \tau+\beta y_{e}(t) r(t), \\
K_{F B}(t) & =K_{0}(t)+K_{\Sigma}(t)+\widehat{K}_{\Sigma}(t), \\
K_{A}(t) & =K_{0 A}(t)+K_{\Sigma A}(t)+\widehat{K}_{\Sigma A}(t)
\end{aligned}
$$


with

$$
\begin{aligned}
& y_{e} \triangleq C_{e} x_{e}, \quad x_{e} \triangleq \widehat{x}-x, \quad C_{e} \triangleq e_{n}^{T} P, \\
& K_{0}(t)=\alpha \int_{0}^{t} y_{e}(\tau) x^{T}(\tau) d \tau+\beta y_{e}(t) x^{T}(t), \\
& K_{\Sigma}(t)=\sum_{j=1}^{M-1} K_{j}(t), \quad \widehat{K}_{\widehat{\Sigma}}(t)=\sum_{\widehat{j}=1}^{\widehat{M}-1} \widehat{K}_{\widehat{j}}(t), \\
& K_{j}(t)=\left\{\begin{array}{l}
\rho \int_{t_{j_{s}}}^{t} y_{e}(\tau) x^{T}(\tau) d \tau, \quad \text { if } \quad x \in \Omega_{j}, \\
0 \quad \text { elsewhere, }
\end{array}\right. \\
& \widehat{K}_{\widehat{j}}(t)=\left\{\begin{array}{l}
\rho \int_{\widehat{t}_{\hat{j}_{s}}}^{t} y_{e}(\tau) x^{T}(\tau) d \tau, \quad \text { if } \quad x \in \widehat{\Omega}_{\widehat{j}}, \\
0 \quad \text { elsewhere, }
\end{array},\right. \\
& K_{0 A}(t)=\rho \int_{0}^{t} y_{e}(\tau) d \tau, \\
& K_{\Sigma A}(t)=\sum_{j=1}^{M-1} K_{A j}, \quad \widehat{K}_{\Sigma A}(t)=\sum_{j=1}^{M-1} \widehat{K}_{A \widehat{j}} \widehat{\sigma}_{\hat{j}} \\
& K_{A j}(t)=\left\{\begin{array}{l}
\rho \int_{t_{j_{s}}}^{t} y_{e}(\tau) d \tau, \quad \text { if } \quad x \in \Omega_{j}, \\
0 \quad \text { elsewhere, }
\end{array}\right. \\
& \widehat{K}_{A \widehat{j}}(t)=\left\{\begin{array}{l}
\rho \int_{\widehat{t}_{\hat{j}_{s}}}^{t} y_{e}(\tau) d \tau, \quad \text { if } \quad x \in \widehat{\Omega}_{\widehat{j}}, \\
0 \quad \text { elsewhere, }
\end{array}\right.
\end{aligned}
$$

and $\alpha, \beta$ and $\rho$ being some positive scalar constants, guarantees that the state tracking error $x_{e}(t)$ between the plant states $x(t)$ in (11) and the reference trajectory $\widehat{x}(t)$ in (3) converges asymptotically to zero, i.e. $\lim _{t \rightarrow \infty} x_{e}(t)=0$.

\section{Remarks}

- The adaptation law presented above consists of three gains $K_{R}, K_{0}$ and $K_{0 A}$ that remain switched on whatever the modes which the plant and reference model are evolving in, together with some gains $K_{j}, \widehat{K}_{\widehat{j}}, K_{A j}$ and $\widehat{K}_{A \hat{j}}$ that are switched on only when the trajectories of the plant or reference model enter certain domains in phase space. Specifically, the switching gains $K_{j}$ and $K_{A j}$ are associated to changes of the mode of the plant, whereas the switching gains $\widehat{K}_{\widehat{j}}$ and $\widehat{K}_{A \widehat{j}}$ are associated to those of the reference model. Furthermore, the gains $K_{R}$ and $K_{0}$ have the same structure of the gains in the Minimal Control Synthesis 
(MCS) approach [15], an application of Landau's Model Reference PI Adaptive Control scheme [16].

- In order to compensate the bounded disturbance acting as an input onto the closed-loop error system, the extended strategy exploits the additional adaptive term $K_{A}(t)$ when compared to the previous version of the algorithm presented in [1], which is a set of switching integral actions used to properly compensate the affine term in each region.

- At the generic $t_{j_{s}}$-th commutation, the adaptive gains $K_{j}$ and $K_{A j}$ are initialized to the last value assumed by that gain when the trajectory of the plant $x(t)$ last exited from region $\Omega_{j}$ (or zero otherwise). Analogously, the adaptive gains $\widehat{K}_{\widehat{j}}$ and $\widehat{K}_{A \widehat{j}}$ at the generic $\widehat{t}_{\widehat{j}_{s}}-$ th commutation, is initialized with the last value assumed by that gain when the trajectory $\widehat{x}(t)$ left the cell $\widehat{\Omega}_{\widehat{j}}$ (or zero otherwise). Hence, according to the notation used for the switching instants, we have:

$$
\begin{aligned}
K_{j}\left(t_{j_{s}}\right) & =K_{j}\left(t_{j_{s-1}+1}\right), s \geq 2 \\
K_{A j}\left(t_{j_{s}}\right) & =K_{A j}\left(t_{j_{s-1}+1}\right), s \geq 2 \\
\widehat{K}_{\widehat{j}}\left(\widehat{t}_{\hat{j}_{s}}\right) & =\widehat{K}_{\widehat{j}}\left(\widehat{t}_{\widehat{j}_{s-1}+1}\right), s \geq 2 \\
\widehat{K}_{A \widehat{j}}\left(\widehat{t}_{\widehat{j}_{s}}\right) & =\widehat{K}_{A \widehat{j}}\left(\widehat{t}_{\widehat{j}_{s-1}+1}\right), s \geq 2 .
\end{aligned}
$$

Note that at the first transition the adaptive gains are set to zero, i.e. $K_{j}\left(t_{j_{1}}\right)=0, K_{j}\left(t_{j_{1}}\right)=0, K_{j}\left(t_{j_{1}}\right)=0, \widehat{K}_{\widehat{j}}\left(\widehat{t}_{\hat{j}_{1}}\right)=0, \widehat{K}_{\widehat{j}}\left(\widehat{t}_{\widehat{j}_{1}}\right)=0$. Furthermore, the integral part of the adaptive gains $K_{R}$ and $K_{0}$ in (17) and (21) are set to zero at time zero.

- Both control gains $K_{R}$ and $K_{0}$ in (17) and (21) have integral and proportional terms. It is worth remarking that the use of integral plus proportional adaptation has a beneficial effect upon the convergence of the generalized state error vector in comparison to the use of integral adaptation, specially at the beginning of the adaptation process [16]. PI adaptation has also been used in [3].

\section{Proof of stability}

We now give the proof of Theorem 1 which is based on constructing an appropriate Common Lyapunov Function (CLF) for the closed-loop system. Note that, due to the presence of discontinuities in the closed-loop system 
dynamics, the error state dynamics $x_{e}(t)$ is evaluated in the sense of Filippov [17] and hence, to prove convergence, the Lyapunov function is also analyzed during possible instances of sliding motion.

As the reference model (3) does not admit sliding solutions by construction, we consider for the switched closed-loop error system the following time-varying domains:

$$
\left\{\Omega_{i}^{c}(t)\right\}_{i \in \mathcal{M}}=\left\{x_{e} \in \mathbb{R}^{n}: \widehat{x}(t)-x_{e} \in \Omega_{i}\right\}_{i \in \mathcal{M}} ;
$$

with $\mathcal{M}=\{0,1, \ldots, M-1\}$. Furthermore we define as $\partial \Omega_{i}^{c}(t)$ their boundaries. (Note that when the error trajectory $x_{e}(t)$ evolves along $\partial \Omega_{i}^{c}(t)$, the plant dynamics exhibit sliding motion along the surface $\partial \Omega_{i}$.)

From definition (33), it is now possible to express the indicator function $\sigma_{i}(t)$ in (12) as a function of the state tracking error as:

$$
\sigma_{i}(t)=\left\{\begin{array}{l}
1 \text { if } x_{e}(t) \in \Omega_{i}^{c}(t) \\
0 \text { elsewhere }
\end{array}\right.
$$

From (23)-(24) and (27)-(28), it trivially follows that, at any given time instant, only one of the pairs of adaptive gains $\left(K_{1} K_{A 1}\right),\left(K_{2} K_{A 2}\right), \ldots,\left(K_{M-1} K_{A M-1}\right)$ and one of the pairs $\left.\left(\widehat{K}_{1} \widehat{K}_{A 1}\right), \widehat{(}_{K_{2}} \widehat{K}_{A 2}\right), \ldots,\left(\widehat{K}_{\widehat{M}-1} \widehat{K}_{A \widehat{M}-1}\right)$ can be different from zero. Hence, equations (22) and (26) can be easily rewritten as:

$$
\begin{aligned}
& K_{\Sigma}(t)=\sum_{j=1}^{M-1} \sigma_{j}(t) K_{j}(t), \quad \widehat{K}_{\widehat{\Sigma}}(t)=\sum_{\widehat{j}=1}^{\widehat{M}-1} \widehat{\sigma}_{\widehat{j}}(t) \widehat{K}_{\widehat{j}}(t), \\
& K_{\Sigma A}(t)=\sum_{j=1}^{M-1} \sigma_{j}(t) K_{A j}, \quad \widehat{K}_{\Sigma A}(t)=\sum_{j=1}^{M-1} \widehat{\sigma}_{\widehat{j}}(t) \widehat{K}_{A \widehat{j}},
\end{aligned}
$$

with $\sigma_{j}$ and $\widehat{\sigma}_{\hat{j}}$ defined as in (34) and (13), respectively.

Given expressions (35), the adaptation law of the control gains (23), (24), (27), (28) can be rewritten in terms of the indicator functions as $(i=1, \ldots, M-1, \widehat{i}=1, \ldots, \widehat{M}-1)$ :

$$
\begin{aligned}
\dot{K}_{i} & =\rho y_{e}(t) x^{T}(t) \sigma_{i}(t), \\
\dot{\widehat{K}}_{\widehat{i}} & =\rho y_{e}(t) x^{T}(t) \widehat{\sigma}_{\widehat{i}}(t), \\
\dot{K}_{A i} & =\rho y_{e}(t) \sigma_{i}(t), \\
\dot{\widehat{K}}_{A \widehat{i}} & =\rho y_{e}(t) \sigma_{\widehat{i}}(t) .
\end{aligned}
$$

Now, from the plant and the reference dynamics given in (11) and (3), respectively, by using the definition of the control strategy in (16), after some algebraic manipulations the dynamics of the state tracking error can be rewritten 
as follows:

$$
\begin{aligned}
& \dot{x}_{e}=\dot{\widehat{x}}-\dot{x}=\widehat{A}_{\widehat{\sigma}(t)} x_{e}+e_{n} \psi_{I} w-e_{n} b \beta y_{e} w^{T} w+ \\
& +e_{n} \sum_{i=1}^{M-1} \sigma_{i} \psi_{i} x+e_{n} \sum_{\widehat{M}-1}^{\widehat{\sigma}^{-1}} \widehat{\sigma}_{\widehat{i}} \widehat{\psi}_{\widehat{i}} x+ \\
& +e_{n}\left[\psi_{A 0}+\sum_{\widehat{i}=1}^{\widehat{M}-1}\left(\widehat{\psi}_{A \hat{i}} \widehat{\sigma}_{\widehat{i}}(t)\right)+\sum_{i=1}^{M-1}\left(\psi_{A i} \sigma_{i}(t)\right)\right],
\end{aligned}
$$

where

$$
\begin{aligned}
& w \triangleq\left[\begin{array}{ll}
x^{T} & r
\end{array}\right]^{T}, \\
& \psi_{I} \triangleq\left[e_{n}^{T}\left(\widehat{A}_{0}-A_{0}\right)-b K_{0}^{I} \quad \vdots \widehat{b}-b K_{R}^{I}\right], \\
& \psi_{i} \triangleq\left[e_{n}^{T} \Delta A_{i}-b K_{i}\right], \Delta A_{i} \triangleq A_{0}-A_{i}, \\
& \widehat{\psi}_{\hat{i}} \triangleq\left[e_{n}^{T} \Delta \widehat{A}_{\widehat{i}}-b \widehat{K}_{\widehat{i}}\right], \Delta \widehat{A}_{\widehat{i}} \triangleq \widehat{A}_{\widehat{i}}-\widehat{A}_{0}, \\
& \psi_{A 0} \triangleq\left(\widehat{b}_{0}-b_{0}\right)-K_{0 A} \text {, } \\
& \widehat{\psi}_{A \widehat{i}} \triangleq \delta \widehat{b}_{\hat{i}}-b \widehat{K}_{A \hat{i}}, \delta \widehat{b}_{\hat{i}} \triangleq\left(\widehat{b}_{\hat{i}}-\widehat{b}_{0}\right) \text {, } \\
& \psi_{A i} \triangleq \delta b_{i}-b K_{A i}, \delta b_{i} \triangleq\left(b_{i}-b_{0}\right) \text {, }
\end{aligned}
$$

with $K_{0}^{I}, K_{R}^{I}$ being the integral part of $K_{0}$ in (21) and $K_{R}$ in (17), respectively, and $i=1, \ldots M-1, \widehat{i}=1, \ldots \widehat{M}-1$. Now, by means of the definition of the adaptive gains given in (17), (21), (25) , (36), (37), (38), (39), the dynamics of $\psi_{I}, \psi_{i}, \widehat{\psi}_{\widehat{i}}, \psi_{A 0}, \psi_{A i}$ and $\widehat{\psi}_{A \hat{i}}$ in (42)-(47) can be written as:

$$
\begin{gathered}
\dot{\psi}_{I}^{T}=-b \alpha y_{e} w, \\
\dot{\psi}_{i}^{T}=-b \rho y_{e} x \sigma_{i}, \\
\dot{\hat{\psi}}_{\hat{i}}^{T}=-b \rho y_{e} x \widehat{\sigma}_{\hat{i}}, \\
\dot{\psi}_{A 0}=-\rho y_{e} b, \\
\dot{\psi}_{A i}=-\rho y_{e} b \sigma_{i}, \\
\dot{\vec{\psi}}_{A \widehat{i}}=-\rho y_{e} b \widehat{\sigma}_{\hat{i}} .
\end{gathered}
$$

Note that, letting $z(t) \in \mathbb{R}^{(n+1)(\widehat{M}+M)-1}$ be the state vector embedding the adaptive gain dynamics (48) as well as the state tracking error (40) the evolution of the closed-loop system (40), (48) can be recast in a more compact form as the following set of differential equations with discontinuous righthand side:

$$
\dot{z}(t)=f_{i}(z) \quad x_{e}(t) \in \Omega_{i}^{c} i=0, \ldots, M-1 .
$$


where $f_{i}$ are the vector fields defined as the right-hand sides of the closedloop system (40), (48).

Now, let $P \widehat{A}_{\widehat{i}}+\widehat{A}_{\widehat{i}}^{T} P=-Q_{\widehat{i}}$ for some $Q_{\widehat{i}}=Q_{\widehat{i}}^{T}>0, \widehat{i} \in \widehat{\mathcal{M}}$ and let $V$ : $\mathbb{R}^{(n+1)(\widehat{M}+M)-1} \rightarrow \mathbb{R}$ be the Lipschitz, regular [18] and positive definite candidate Lyapunov function given by:

$$
\begin{aligned}
& V=x_{e}^{T} P x_{e}+\frac{1}{\alpha b} \psi_{I} \psi_{I}^{T}+\frac{1}{\rho b} \sum_{i=1}^{M-1} \psi_{i} \psi_{i}^{T}+\frac{1}{\rho b} \sum_{\widehat{i}=1}^{\widehat{M}-1} \widehat{\psi}_{\hat{i}} \widehat{\psi}_{\widehat{i}}^{T}+ \\
& +\frac{1}{\rho b} \psi_{A 0}^{2}+\frac{1}{\rho b} \sum_{i=1}^{M-1} \psi_{A i}^{2}+\frac{1}{\rho b} \sum_{\hat{i}=1}^{\widehat{M}-1} \widehat{\psi}_{A i}^{2} .
\end{aligned}
$$

To prove asymptotic stability of (49) in what follows we will first evaluate of $\dot{V}$ in the interior of each generic region $\Omega_{i}^{c}$ and then along the generic surfaces $\partial S_{l}$ resulting from intersections of the manifolds $\partial \Omega_{i}^{c}$ (here $l=1, \ldots, L ; L$ being the number of manifolds where sliding is possible) [19]:

$$
\partial S_{l}=\bigcap_{d=1}^{H} \partial \Omega_{i_{d}}^{c},
$$

with $H \leq(M-1)$.

\subsubsection{Evaluation of $\dot{V}$ in $\Omega_{i}^{c}$}

In the interior of each region $x_{e}(t) \in \Omega_{i}^{c}$, the error system (49) is a smooth set of differential equations composed by equations (40), (48) with $\sigma$ and $\widehat{\sigma}$ taking finite constant values associated to the active modes of the plant and reference model in that region. The time derivative of $V$ along the trajectories (401) can be computed as:

$$
\begin{aligned}
& \dot{V}=-x_{e}^{T} Q_{\widehat{\sigma}(t)} x_{e}+\frac{2}{\alpha b} \psi_{I} \dot{\psi}_{I}^{T}+\frac{2}{\rho b} \sum_{i=1}^{M-1} \psi_{i} \dot{\psi}_{i}^{T}+\frac{2}{\rho b} \sum_{\widehat{i}=1}^{\widehat{M}-1} \widehat{\psi}_{\hat{\imath}} \dot{\psi}_{\widehat{i}}^{T}+ \\
& +2 x_{e}^{T} P\left[e_{n} \psi_{I} w-e_{n} b \beta y_{e} w^{T} w+e_{n} \sum_{i=1}^{M-1} \sigma_{i} \psi_{i} x+e_{n} \sum_{\widehat{M}-1}^{\widehat{M}-1} \widehat{\sigma}_{\widehat{i}} \widehat{\psi}_{\hat{i}} x\right]+ \\
& +2 x_{e}^{T} P e_{n}\left[\psi_{A 0}+\sum_{i=1}^{M-1}\left(\psi_{A i} \sigma_{i}(t)\right)+\sum_{\widehat{i}=1}^{\widehat{M}-1}\left(\widehat{\psi}_{A \widehat{i}} \widehat{\sigma}_{\widehat{i}}(t)\right)\right]+ \\
& \frac{2}{\rho b} \psi_{A 0} \dot{\psi}_{A 0}+\frac{2}{\rho b} \sum_{i=1}^{M-1} \psi_{A i} \dot{\psi}_{A i}+\frac{2}{\rho b} \sum_{\widehat{i}=1}^{\widehat{M}-1} \widehat{\psi}_{A \hat{i}} \dot{\hat{\psi}}_{A \widehat{i}} .
\end{aligned}
$$

Substituting (48a)-(48f) into equation (52) and taking into account that $x_{e}^{T} P e_{n}=e_{n}^{T} P x_{e}=y_{e}$, after some algebraic manipulations we have:

$$
\begin{aligned}
\dot{V}=-x_{e}^{T} Q_{\widehat{\sigma}} x_{e}-2 b \beta y_{e}^{2} w^{T} w \leq \\
\quad-\min _{\hat{i} \in \widehat{\mathcal{M}}}\left(\lambda_{\min }\left[Q_{\hat{i}}\right]\right)\left\|x_{e}\right\|^{2}=-W\left(x_{e}\right),
\end{aligned}
$$


where $\lambda_{\min }\left[Q_{\hat{i}}\right]$ is the smallest eigenvalue of $Q_{\hat{i}}$.

\subsubsection{Evaluation of $\dot{V}$ along the manifolds $\partial S_{l}$}

In this case two different situations may occur; (i) the trajectory $x_{e}(t)$ crosses the generic manifold $\partial S_{l}$ (51) over a time interval of zero Lebesgue measure, or (ii) it exhibits sliding solutions. In the former case, the crossing has no effect on the stability analysis. Therefore, we focus below on the case where sliding occurs.

In particular, when sliding takes place, solutions should be interpreted in the sense of Filippov [17]. Using Filippov convex method, we consider the sliding vector field, say $f_{F}$, obtained by the convex combination [20]:

$$
f_{F}:=\sum_{i=0}^{M-1} f_{i}(z) \gamma_{i}(z), \quad \text { with } \gamma_{i}(z) \geq 0
$$

where $f_{i}$ are the vector fields defined in equation (49) and $\sum_{i=0}^{M-1} \gamma_{i}(z)=1$. Note that in the general case this is an underdetermined system of equations, hence there is no uniquely defined Filippov sliding vector. Since the following stability analysis does not depend on any particular choice of Filippov vector field, we do not consider this issue.

From (54), after some algebraic manipulations it is possible to write the closed-loop dynamics (40), (48) during the sliding motion as:

$$
\begin{gathered}
\dot{x}_{e}=\widehat{A}_{\widehat{\sigma}(t)} x_{e}+e_{n} \psi_{I} w-e_{n} b \beta y_{e} w^{T} w+e_{n} \sum_{\widehat{i}=1}^{\widehat{M}-1} \widehat{\sigma}_{\widehat{i}} \widehat{\psi}_{\hat{i}} x \\
+e_{n} \sum_{\widehat{i}=1}^{\widehat{M}-1} \widehat{\sigma}_{\widehat{i}} \widehat{\psi}_{A \hat{i}}+e_{n} \psi_{A 0}+e_{n} \sum_{i=1}^{M-1} \gamma_{i}\left(\psi_{i} x+\psi_{A i}\right) \\
\dot{\psi}_{I}^{T}=-b \alpha y_{e} w \\
\dot{\psi}_{i}^{T}=-\gamma_{i} b \rho y_{e} x, \\
\dot{\hat{\psi}}_{\widehat{i}}^{T}=-b \rho y_{e} x \widehat{\sigma}_{\widehat{i}}, \\
\dot{\psi}_{A 0}=-\rho y_{e} b, \\
\dot{\psi}_{A i}=-\gamma_{i} b \rho y_{e}, \\
\dot{\hat{\psi}}_{A \hat{i}}=-\rho y_{e} b \widehat{\sigma}_{\hat{i}} .
\end{gathered}
$$

where only quantities depending on discontinuities due to plant switchings are convexified as the reference model is assumed not to exhibit sliding. 
Evaluating the derivative of $V$ (50) (again in the sense of Filippov) along trajectories of (55), some algebraic manipulations yield:

$$
\dot{V} \leq-\min _{\widehat{i} \in \widehat{\mathcal{M}}}\left(\lambda_{\min }\left[Q_{\hat{i}}\right]\right)\left\|x_{e}\right\|^{2}=-W\left(x_{e}\right) .
$$

\subsubsection{Stability of the closed-loop adaptive system}

From (53) and (56), it follows that for almost all $t$

$$
\dot{V} \leq-W\left(x_{e}\right)<0 \text { a.e. } t, \forall x_{e} \forall \psi_{I}, \psi_{i}, \widehat{\psi}_{\hat{i}}, \psi_{A 0}, \psi_{A i}, \widehat{\psi}_{A \hat{i}} .
$$

The derivative of the Lyapunov function along Filippov closed-loop solutions is negative, hence the origin of the closed-loop system is globally stable in the Filippov sense [18].

Now following the approach in [21], from (57) for any closed-loop trajectory we have

$$
\sup _{t \in[0+\infty)} V \leq C \quad \forall t \geq 0
$$

with $C$ being a sufficient large positive constant.

From (57) and (58), it follows

$$
\int_{0}^{\infty} W\left(x_{e}(t)\right) d t \leq C
$$

Since $W\left(x_{e}\right)$ is a continuously differentiable positive-definite function, $W\left(x_{e}(t)\right)$ is uniformly continuos. Exploiting Barbalat's Lemma, $W\left(x_{e}(t)\right)$ converges to 0 as $t \rightarrow \infty$, hence the state tracking error $x_{e}(t)$ converges to 0 .

\section{Conclusions}

We have presented an extension of the hybrid adaptive strategy introduced in [1] aimed at compensating possible instabilities due to the presence of sliding mode trajectories and bounded perturbations acting on the closed loop error system. The external disturbances are due to the presence of affine terms in the plant and reference model dynamics. Using an appropriate common Lyapunov function, we have shown that the extended strategy guarantees asymptotic convergence of the tracking error, even in the presence of sliding solutions, as well as boundedness of all the adaptive gains. 


\section{References}

[1] M. di Bernardo, U. Montanaro, and S. Santini. Hybrid model reference adaptive control of piecewise affine systems. IEEE Transactions on Automatic Control, 58(2):304-316, 2013.

[2] Gang Tao. Multivariable adaptive control: A survey. Automatica, 50(11):2737-2764, 2014.

[3] A. Astolfi, D. Karagiannis, and R. Ortega. Nonlinear and Adaptive Control with Applications. Springer-Verlag, 2007.

[4] B. D. O. Anderson. Failures of adaptive control theory and their resolution. Communications in Information and Systems, 5:1620, 2005.

[5] B. D. O. Anderson, R. R. Bitmead, C. R. Johnson, P. V. Kokotovic, R. L. Kosut, I. M. Y. Mareels, L. Praly, and B. D. Riedle. Stability of Adaptive Systems: Passivity and Averaging Analysis. The M.I.T. Press, Cambridge, MA and London, 1986.

[6] L. Wang, A. Chaillet, and R. Ortega. Personal communication, 2013.

[7] M. di Bernardo, U. Montanaro, and S. Santini. Minimal control synthesis adaptive control of continuous bimodal piecewise affine systems. SIAM Journal on Control and Optimization, 48(7):4242-4261, 2010.

[8] M. di Bernardo, U. Montanaro, and S. Santini. Novel switched model reference adaptive control for continuous piecewise affine systems. Proc. of the 47th IEEE Conference on Decision and Control, pages 1925-1930, 2008.

[9] Mario di Bernardo, Carlos Ildefonso Hoyos Velasco, Umberto Montanaro, and Stefania Santini. Experimental implementation and validation of a novel minimal control synthesis adaptive controller for continuous bimodal piecewise affine systems. Control Engineering Practice, $20(3): 269-281,2012$.

[10] M. di Bernardo, U. Montanaro, J.O. Olm, and S. Santini. Model reference adaptive control of discrete-time piecewise linear systems. International Journal of Robust and Nonlinear Control, 23(7):709-730, 2013.

[11] Mario di Bernardo, Alessandro di Gaeta, Umberto Montanaro, Josep M. Olm, and Stefania Santini. Experimental validation of the discrete-time MCS adaptive strategy. Control Engineering Practice, 21(6):847 - 859, 2013. 
[12] M. di Bernardo U., Montanaro, and S. Santini. Canonical forms of generic piecewise linear continuous systems. IEEE Transactions on Automatic Control, 56(8):1911 -1915, aug. 2011.

[13] M. S. Branicky. Multiple lyapunov functions and other analysis tools for switched and hybrid systems. IEEE Transactions on Automatic Control, 43(4):475-482, 1998.

[14] J. Zhao and D. J. Hill. Dissipativity theory for switched systems. IEEE Transactions on Automatic Control, 53(4):941-953, 2008.

[15] S.P. Hodgson and D. P. Stoten. Passivity-based analysis of the minimal control synthesis algorithm. International Journal of Control, 63(1):6784, 1996.

[16] I.D. Landau. Adaptive Control: the model reference approach. SpringerVarlag, 1979.

[17] A.F. Filippov. Differential Equations with Discontinuous Righthand Sides: Control Systems (Originally published in Russian), volume 18 of Mathematics and its Applications. Springer, 1988.

[18] D. Shevitz and B. Paden. Lyapunov stability theory of nonsmooth systems. Automatic Control, IEEE Transactions on, 39(9):1910-1914, Sep 1994.

[19] James C. Alexander and Thomas I. Seidman. Sliding modes in intersecting switching surfaces. Huston Journal of Mathematics, 24(3):545-569, 1998.

[20] Luca Dieci and Luciano Lopez. Sliding motion on discontinuity surfaces of high co-dimension. a construction for selecting a filippov vector field. Numerische Mathematik, 117(4):779-811, 2011.

[21] Takashi Nakakuki, Tielong Shen, and Katsutoshi Tamura. Adaptive control design for a class of nonsmooth nonlinear systems with matched and linearly parameterized uncertainty. International Journal of Robust and Nonlinear Control, 19(2):243-255, 2009. 